\title{
Adipose-derived mesenchymal stem cell-facilitated TRAIL expression in melanoma treatment in vitro
}

\author{
HAI XIA JING ${ }^{1}$, DE JIAN DUAN ${ }^{2}$, HUI ZHOU ${ }^{2}$, QING MEI HU ${ }^{1}$ and TIE CHI LEI ${ }^{1}$ \\ ${ }^{1}$ Department of Dermatology, Renmin Hospital of Wuhan University, Wuhan, Hubei 430060; \\ ${ }^{2}$ Department of Dermatology, Taihe Hospital, Hubei University of Medicine, Shiyan, Hubei 442000, P.R. China
}

Received April 20, 2015; Accepted February 29, 2016

DOI: $10.3892 / \mathrm{mmr} .2016 .5283$

\begin{abstract}
Adipose-derived stem cells (ADSCs) may be useful as an efficient vehicle in cell-based gene therapy of human diseases due to their ability to migrate to disease lesions. This study investigated the ability of ADSC-harbored human tumor necrosis factor-related apoptosis-inducing ligand (TRAIL) cDNA to facilitate TRAIL expression and induce A375 melanoma cell apoptosis as observed using a Transwell co-culture system. A cell migration assay was used to observe ADSC migration ability. In addition, TRAIL protein expression was successfully detected by western blot analysis in ADSCs after stable transfection of TRAIL cDNA. The Transwell co-culture system data showed that TRAIL-ADSCs could induce A375 cell apoptosis in a dose-dependent manner. At the gene level, the killing activity of TRAIL-ADSCs was associated with activation of caspase- 4 and caspase-8. Collectively, the data from the current study provides preclinical support of ADSC-facilitated TRAIL expression in the treatment of melanoma. Further investigation is required to evaluate and confirm the in vivo ability of TRAIL-ADSCs in therapy of melanoma in animal models.
\end{abstract}

\section{Introduction}

Malignant melanoma is an aggressive skin cancer that arises from the melanocyte lineage (1). Melanoma occurs less frequently than the majority of other malignancies, accounting for 232,000 new cases and resulting in 55,000 cancer-related mortalities worldwide in 2012 (2). However, it is dangerous if diagnosed at the later stages of the disease and causes the majority of skin cancer-related fatalities (3). Melanoma is usually caused by DNA damage resulting from exposure to ultraviolet light from sun exposure, or genetic predisposition (4).

Correspondence to: Professor Tie Chi Lei, Department of Dermatology, Renmin Hospital of Wuhan University, 95 Zhang Zhidong Road, Wuhan, Hubei 430060, P.R. China

E-mail: tchlei@whu.edu.cn

Key words: gene therapy, melanoma, adipose derived stem cell, tumor necrosis factor-related apoptosis-inducing ligand
Early stages of melanoma are curable by surgical removal of the tumor lesion, while later stages or recurrent melanoma are treated with chemo- and immunotherapy, or radiation therapy $(5,6)$. However, to date, melanoma is one of the most difficult types of cancer to successfully treat as it disseminates early after the development of the initial lesion. The majority of all melanoma patients with advanced stage disease succumb to distant metastases 6-10 months after initial diagnosis (7). The major obstacle in melanoma treatment is the lack of tumor specificity. Thus, more efficient treatment strategies specifically targeting tumor tissue are urgently required (8).

For targeted therapy of melanoma, like the majority of other cancer types, mesenchymal stem cells (MSCs) may have tumor-oriented homing capacity and thus potential as an efficient vehicle in patient-tailored cancer therapy $(9,10)$. Tumor-directed migration and incorporation of MSCs have been demonstrated in a number of pre-clinical studies using in vitro and in vivo animal tumor models $(8,11,12)$. For example, bone marrow mesenchymal stem cells (11), human pancreas stem cells (8) and neural stem cells (12) have the capability of tumor tracking and this tracking capability was associated with the secretion of cytokines and chemokines. The stem cells can migrate to and gather around the tumor lesion with a high concentration and this feature suggested that MSCs could be used as a carrier of enzyme/prodrug gene in combined targeting therapy of human cancers. The homing capacity of MSCs has previously been demonstrated in almost all tested human cancer cell lines, including melanoma (13). Bone marrow (BM) was the first recognized source of MSCs (14); however, adipose tissue represents a more reliable source of MSCs (15). Compared with BM-MSCs, adipose-derived MSCs (ADSCs) are more suitable for tumor-gene therapy approaches. This is because adipose tissue can be obtained in relevant quantities by minimally invasive procedures from normal subjects or from cancer patients $(16,17)$.

Furthermore, tumor necrosis factor (TNF)-related apoptosis-inducing ligand (TRAIL) is a promising anticancer death ligand with sequence homology to TNF and FasL (18). TRAIL is one of few anticancer proteins that can selectively induce apoptosis of transformed or tumor cells by activation of death receptors (DR), without affecting healthy cells (19). In previous in vitro experiments, TRAIL was shown to induce apoptosis of glioma, neuroblastoma, cervix uteri cancer, non-small cell lung cancer, renal cell carcinoma, liver cancer, thyroid cancer 
and melanoma cells. In addition, it was shown to exhibit a particularly lethal effect on lung cancer cells (13), malignant glioma cells (1) and breast cancer cells (20). A previous study also showed that TRAIL significantly inhibited the growth of hepatocellular carcinoma cells in mice, but did not exhibit any toxic side effects on the control mice (21). Thus, several studies demonstrated the antitumor activity of recombinant TRAIL (rTRAIL), but rTRAIL in vivo use is limited due to its short half-life in the blood (22). It has been reported that ADSCs could be used to deliver a stable source of TRAIL for cancer therapy (23). Thus, the current study utilized ADSCs to harbor TRAIL cDNA to facilitate TRAIL expression and test the in vitro effects on melanoma cells.

\section{Materials and methods}

Cell lines and culture. Human ADSCs (HUXMD-01001) were purchased from Cyagen Biotechnology Co., Ltd. (Guangzhou, China) and cultured in Dulbecco's modified Eagle's medium (DMEM)-F12 supplemented with $10 \%$ Gibco fetal bovine serum (FBS; \#16000044), 2 mM L-glutamine, and 1\% penicillin-streptomycin solution (Thermo Fisher Scientific, Inc., Waltham, MA, USA) in a humidified incubator with $5 \% \mathrm{CO}_{2}$ at $37^{\circ} \mathrm{C}$. The immunophenotype identification of ADSCs was tested by flow cytometry. Adipogenic and osteogenic differentiation of ADSCs was conducted using cell differentiation kits (HUXMD-90031 and -90021; Cyagen Biotechnology Co., Ltd.). The constituents of the adipogenic induction medium A were high-glucose DMEM supplemented with $10 \% \mathrm{FBS}$, $2 \mathrm{mM}$ L-glutamine, 100 units myllicin, $5 \mu \mathrm{g} / \mathrm{ml}$ insulin, $0.5 \mu \mathrm{M} 3$-isobutyl-1-methylxantine, rosiglitazone and $10 \mathrm{nM}$ dexamethasone (kit HUXMD-90031). Adipogenic induction medium B contained high-glucose DMEM supplemented with $10 \%$ FBS, 2 mM L-glutamine, 100 units myllicin and $5 \mu \mathrm{g} / \mathrm{ml}$ insulin (kit HUXMD-90031). Osteogenic induction medium contained high-glucose DMEM supplemented with $10 \% \mathrm{FBS}$, $2 \mathrm{mM}$ L-glutamine, 100 units myllicin, $100 \mu \mathrm{g} / \mathrm{ml}$ ascorbate, $10 \mathrm{mM} \beta$-glycerophosphate and $100 \mathrm{nM}$ dexamethasone (kit HUXMD-90021). After 14-20 days induction, the differentiated cells were fixed with $70 \%$ ethanol, then washed 3 times with PBS and stained with Alizarin red (kit HUXMD-90021), or Oil Red O (kit HUXMD-90031) according to the manufacturer's instructions. The A375 human melanoma cell line was obtained from the American Type Culture Collection (Manassas, VA, USA) and was cultured according to the manufacturer's instructions. Briefly, A375 cells were cultured in DMEM supplemented with $10 \%$ FBS and 100 units myllicin, in a humidified incubator with $5 \% \mathrm{CO}_{2}$ at $37^{\circ} \mathrm{C}$.

Construction of TRAIL-carrying vector and gene transfection. Full-length human TRAIL cDNA was cloned into the pcDNA3.3-TOPO plasmid using a pcDNA 3.4 TOPO TA Cloning Kit (\#A14697; Thermo Fisher Scientific, Inc.) as shown in Fig. 1A. In brief, TRAIL cDNA was amplified from MIGR1-TRAIL-GFP as described by Wiley et al (18) and then sub-cloned into the pcDNA3.3-TOPO plasmid. The TRAIL cDNA was connected to the pcDNA 3.3-TOPO plasmid with T4 DNA ligase (\#D7006; Beyotime Institute of Biotechnology, Beijing, China). After colony polymerase chain reaction (PCR) amplification (the template was bacterium suspension) and DNA sequencing confirmation (performed by Sangon Biotech Co., Ltd., Wuhan, China), this plasmid containing TRAIL cDNA was used for tail PCR and modified with the MEGAscript T7 kit (Ambion; Thermo Fisher Scientific, Inc.). Then, modified TRAIL mRNA was isolated with Ambion Anti-Reverse Cap Analog (ARCA; \#AM8045) and purified with Ambion MEGAclear spin columns (Thermo Fisher Scientific, Inc.) and treated with Antarctic Phosphatase (New England Biolabs, Ipswich, MA, USA) to remove residual 5'-triphosphates. The transfection of the TRAIL plasmid into ADSCs was conducted using TransIT-mRNA (Mirus Bio LLC., Madison, WI, USA) according to the manufacturer' instructions and ADSCs transfected with TRAIL-cDNA were defined as TRAIL-ADSCs.

Immunofluorescence. Initially, $1 \times 10^{5}$ human foreskin fibroblast (HFF) cells from our laboratory stocks were seeded onto coverslips and grown overnight, and then fixed with $4 \%$ paraformaldehyde for $30 \mathrm{~min}$. For immunofluorescence, the cells were first incubated with $3 \% \mathrm{H}_{2} \mathrm{O}_{2}$ in phosphate-buffered saline (PBS) solution for $30 \mathrm{~min}$ and then washed with tap water and again with PBS. Next, the cells were incubated with 5\% normal goat serum (\#C1771; Applygen Technologies, Inc., Beijing, China) in PBS for $30 \mathrm{~min}$, then incubated with a polyclonal mouse vimentin antibody (1:200; \#3390S; Cell Signaling Technology, Inc., Danvers, MA, USA) and then a secondary fluorescein isothiocyanate (FITC)-conjugated goat anti-mouse IgG (1:500; \#A0568; Beyotime Institute of Biotechnology), and the nuclei were stained with RedDot 1 (\#40060-T; Biotium, Hayward, CA, USA). In addition, $1 \times 10^{5}$ cells were seeded into 24-well plates and cocultured with ADSCAs or ADSCs/TRAIL in Transwell chambers. The dead cells were stained with propidium iodide (PI; \#P4170; Sigma-Aldrich, St. Louis, MO, USA) and then reviewed and scored under a DM IRE2 fluorescent microscope (Leica Microsystems GmbH, Wetzlar, Germany). The dead cells were shown by red fluorescence staining and the live cells did not stain red.

Transwell cell migration assay. To assess the migration ability of ADSCs, a Transwell migration assay using a Transwell system from Corning (Corning, Inc., Corning, NY, USA) was performed. Specifically, A375 cells were seeded onto the plastic surface of the 24-well plates at a density of $1 \times 10^{5}$ cells/well and grown overnight, while HFF cells were used as a control. On the following day, parental ADSCs and TRAIL-ADSCs were seeded on the top chambers of Transwells, respectively and cultured in serum-free medium for $48 \mathrm{~h}$ at $37^{\circ} \mathrm{C}$ and in $5 \% \mathrm{CO}_{2}$ in an incubator. At the end of the experiments, the up-chamber was washed with PBS and scraped gently using a cotton swab to remove non-migrated cells. The ADSCs migrated to the bottom side of the filter and were fixed with $70 \%$ ethanol, stained with $0.1 \%$ crystal violet (\#C0121; Beyotime Institute of Biotechnology) and counted under a DMI3000 M inverted manual microscope (Leica Microsystems $\mathrm{GmbH}$ ). The average number of migrated cells was assessed by counting five randomly selected microscopic fields. The experiment was performed in triplicate.

Flow cytometry cell apoptosis assay. To assess the apoptosis-induction effects of ADSC-TRAIL on A375 cells, cells 
were cultured under direct co-culture conditions. In brief, A375 cells and TRAIL-ADSCs were plated at 1:1, 1:2 or 1:5 ratios in 6-well plates and were cultured for up to $48 \mathrm{~h}$. At the end of the experiments, the cells were stained with PI and analyzed using an EPICS XL flow cytometer (Beckman Coulter, Danvers, MA, USA). Soluble hrTRAIL (PeproTech, Inc., London, UK) was used as a positive control. Anti-human TRAIL antibody diluted at different concentrations was used to neutralize the TRAIL-induced apoptosis, which was demonstrated in co-culture of A375 cells and TRAIL-ADSCs at a 1:1 ratio. The experiment was performed in triplicate.

Protein extraction and western blotting. Western blotting was performed to detect the cellular expression of TRAIL protein in the ADSCs and apoptosis-related proteins (caspase-3, caspase-4, caspase- 8 and caspase-9) in A375 cells. A375 cells and ADSCs were co-cultured in a Transwell system for $48 \mathrm{~h}$ and then, ADSCs were collected. Equal amounts of whole cell lysates were resolved by $12 \%$ SDS-PAGE and electrotransferred onto a polyvinylidene difluoride membrane (Beyotime Institute of Biotechnology). The PVDF membranes were blotted with $5 \%$ BSA and then incubated with primary antibodies, including monoclonal mouse anti-human STRAIL (1:500; \#500-M49; PeproTech, Rocky Hill, NJ, USA), polyclonal rabbit anti-human caspase-4 (1:200; \#4450S; Cell Signaling Technology, Inc.), caspase-3 (1:500; \#AC030), caspase-8 (1:500; \#AC056), caspase-9 (1:500; AC062), Akt (1:500; \#AA326), phospho-Akt (Ser473; 1:500; \#AA329) and $\beta$-actin (1:1,000; AA128) antibodies (Beyotime Institute of Biotechnology). After washing 3 times with Tris-buffered saline with Tween 20, the PVDF membranes were incubated with alkaline phosphatase-labeled goat anti-rabbit $(1: 1,000$; \#A0239) or anti-mouse (1:1,000; \#A0258) IgGs (Beyotime Institute of Biotechnology). The immunoreactive signals were detected using a Gel Docx2000 scanner system (Bio-Rad Laboratories, Inc., Hercules, CA, USA) and analyzed with Image J software, version 2.1.4.7 (imagej.nih.gov/ij).

Enzyme-linked immunosorbent assay (ELISA). The level of TRAIL protein in the cell-conditioned medium was measured using a Quantikine Human TRAIL/TNFSF10 kit (R\&D Systems, Minneapolis, MN, USA) according to the manufacturer's instructions. Briefly, the cell-conditioned medium from the Transwell system was collected and centrifuged at $206 \mathrm{x} g$ for $3 \mathrm{~min}$ to remove cell debris and the supernatant was subjected to ELISA. The experiments were performed in triplicate and repeated three times.

Statistical analysis. Measurement data are expressed as the mean \pm standard error. Statistical differences between the means of the different groups were evaluated using SPSS 18.0 software (Chicago, IL, USA) using two-tailed Student's t-test. $\mathrm{P} \leq 0.05$ was considered to indicate a statistically significant difference.

\section{Results}

Expression of TRAIL protein in ADSCs. TRAIL-containing plasmids were transiently transfected into ADSCs and western blot and ELISA analyses showed expression of TRAIL protein
A

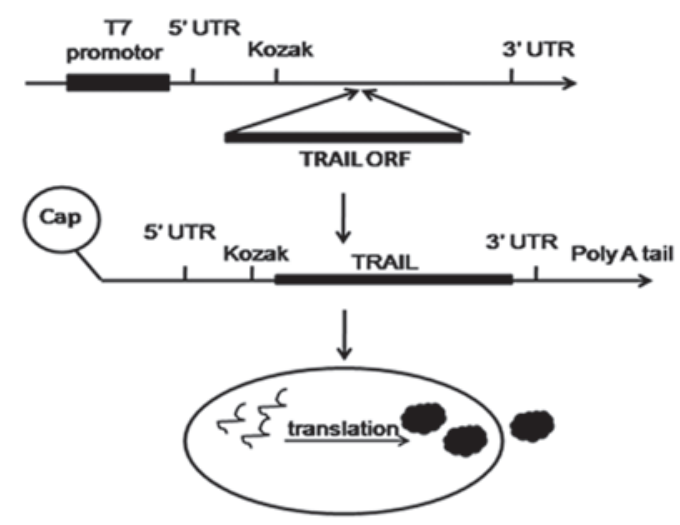

B
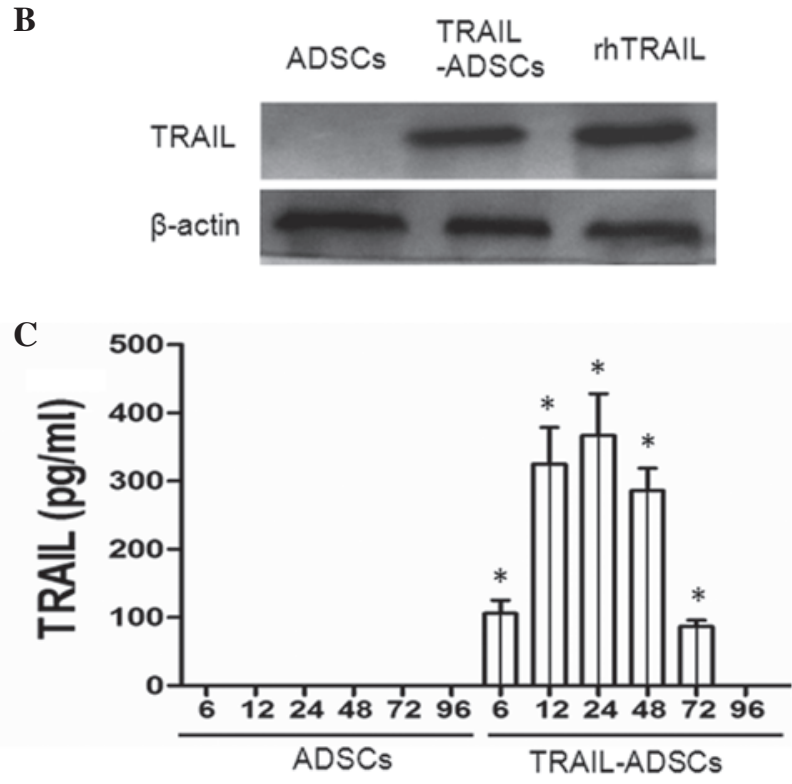

Figure 1. TRAIL construct and expression in ADSCs. (A) Schematic representation of the TRAIL construct and modification. (B) The TRAIL construct was transfected into ADSCs and cells were harvested 2 days later and subjected to western blotting. (C) Enzyme-linked immunosorbent assay analysis of the half-life of sTRAIL in the conditioned medium. Culture medium from ADSCs and TRAIL-ADSCs was collected at different time points after gene transfection. "P $<0.05$ vs. ADSCs. TRAIL, tumor necrosis factor-related apoptosis-inducing ligand; ADSCs, adipose-derived stem cells.

was clearly enhanced in transfected ADSCs (Fig. 1B) and in the conditioned medium (Fig. 1C) in a time-dependent manner. Specifically, soluble TRAIL could be detected after gene transfection at $6 \mathrm{~h}(126.8 \pm 18.4 \mathrm{pg} / \mathrm{ml})$, the levels increased at $12 \mathrm{~h}$ $(325.1 \pm 53.6 \mathrm{pg} / \mathrm{ml})$ and peaked at $24 \mathrm{~h}(366.4 \pm 57.5 \mathrm{pg} / \mathrm{ml})$ and reduced at $96 \mathrm{~h}$ (Fig. 1C), suggesting that the TRAIL mRNA was synthesized effectively.

Characteristics of ADSC differentiation in vitro. Firstly, the properties of ADSCs and A375 cells in vitro were characterized, while HFF cells, which have long spindle morphology and are vimentin-positive, were used as a control (Fig. 2A and B). The ADSCs used in the present study exhibited positive expression of $\mathrm{CD}_{4} 4^{+}, \mathrm{CD}^{2} 3^{+}, \mathrm{CD} 29^{+}$and were negative for CD31-, CD34 and $\mathrm{CD}^{-} 5^{-}$(Fig. 2C). ADSC differentiation was induced using the different kit that could induce ADSCs differentiated into adipogenic and osteogenic cells under specific differentiation 

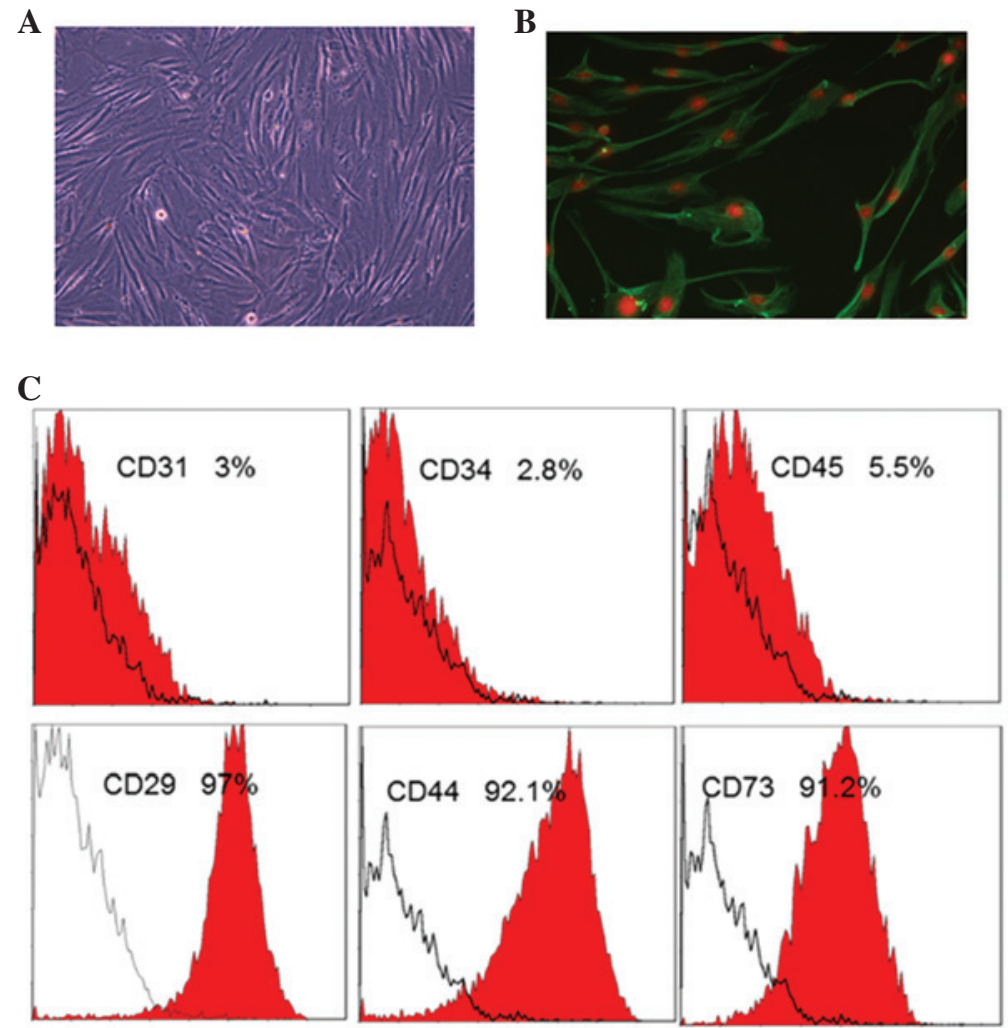

D

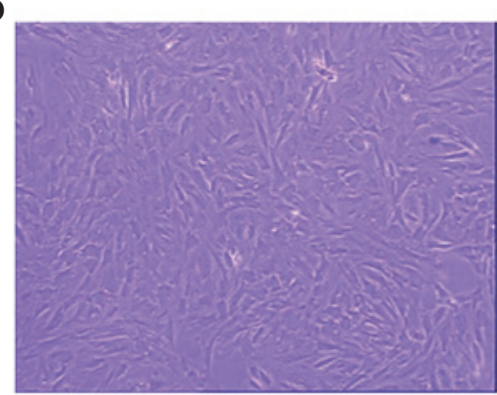

$\mathbf{E}$

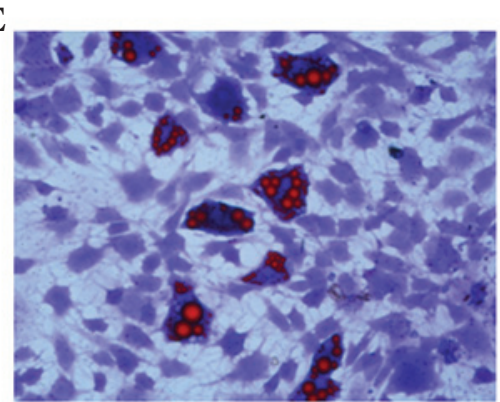

$\mathbf{F}$

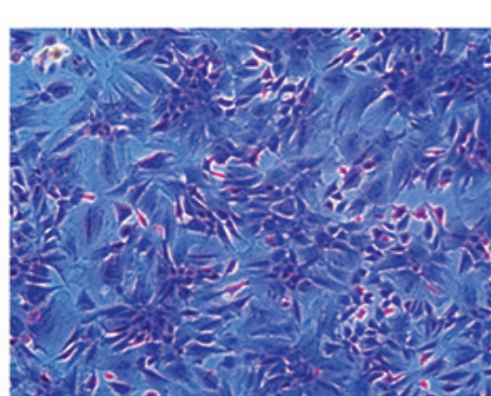

Figure 2. Characteristics of HFFs and ADSCs. (A) In vitro culture of HFFs at day 3. (B) Immunofluorescence analysis of vimentin expression (green) in HFFs. (C) The immunophenotype identification of ADSCs. (D) Morphology of ADSCs under phase control. (E) ADSCs were cultured and induced by a differentiation kit for 10 days and subjected to Oil Red staining (the positive cells were adipose cells). (F) ADSCs were cultured and induced by a differentiation kit for 14 days, ADSCs differentiated into Alizarin Red-positive osteocytes cells. Magnification, x100. TRAIL, tumor necrosis factor-related apoptosis-inducing ligand; HFFs, human foreskin fibroblast; ADSCs, adipose-derived stem cells.

conditions (Fig. 2D-F). Specifically, when cultured in adipogenic differentiation medium for 10-14 days, there were numerous lipid cells with big fat droplets positive for oil red staining (Fig. 2E). By contrast, following culture in osteogenic differentiation medium for 14-21 days, there were numerous osteoblast cells positive for Alizarin red staining (Fig. 2F).

Effects of melanoma cells on the regulation of ADSC migration. ADSC migration was determined by a Transwell assay and HFF cells were used as a control. After co-culture for $48 \mathrm{~h}$, a considerable number of ADSCs migrated across the Transwell membrane (Fig. 3A). ADSCs and TRAIL-ADSCs exhibited significantly stronger migration ability towards A375 melanoma cells (123 \pm 15 and $131 \pm 16$, respectively) compared with HFF cells $(51 \pm 6$ and $53 \pm 5$, respectively) $(\mathrm{P}<0.05)$. Compared with the control ADSCs, TRAIL cDNA transfection did not obstruct ADSC migration towards A375 cells (Fig. 3A and B).
These results suggest that ADSCs and TRAIL-ADSCs have a specific migration ability toward tumor cells, and the transfection of TRAIL mRNA did not affect this ability.

Effects of TRAIL expression facilitated by ADSCs on the regulation of melanoma cell viability. Indirect co-culture was used to determine the effects of TRAIL-ADSCs on the viability of A375 cells using PI staining. As shown in Fig. 4A, TRAIL-ADSCs induced A375 cell death, which was represented by a reduction in the number of adherent A375 cells and the presence of cellular debris. These features were even more prominent after $48 \mathrm{~h}$ of co-culture. However, the parental ADSCs did not exhibit this effect. The PI-positive dead A375 cells were observed in the TRAIL-ADSCs group (Fig. 4A). As shown in Fig. 4B, apoptosis of A375 cells was only detectable in co-culture with TRAIL-ADSCs starting from the $1: 1$ ratio $(35.2 \pm 3.3, \mathrm{P}<0.01)$. This effect increased signifi- 
A

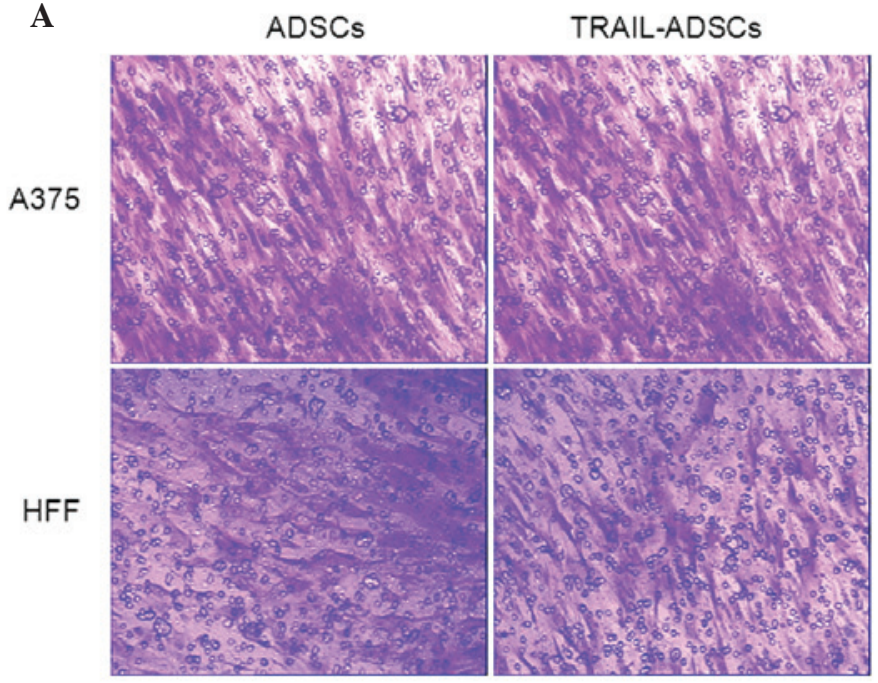

B

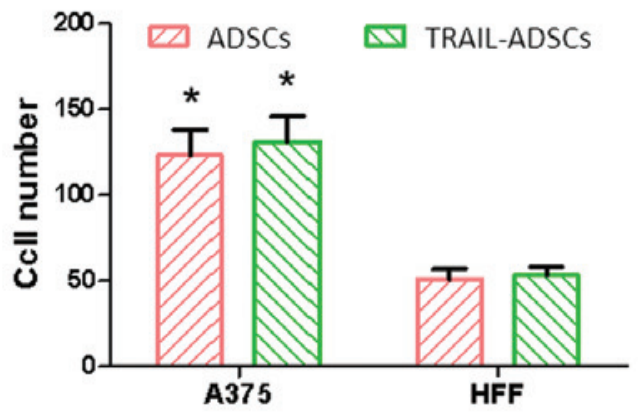

Figure 3. In vitro migratory ability of ADSCs. (A) Parental ADSCs, TRAIL-ADSCs or HFFs were co-cultured with A375 in a Transwell system for 24 days and the migrated cells were stained with crystal violet. Magnification, x100. (B) Quantification of data. Values were from three independent experiments and were presented as the mean \pm standard error of the mean. The cell number of each filter membrane was obtained from an average of five randomly selected microscopic fields. "P<0.05 vs. HFF. TRAIL, tumor necrosis factor-related apoptosis-inducing ligand; HFFs, human foreskin fibroblast; ADSCs, adipose-derived stem cells.

cantly as the ratio of ADSCs increased: Tumor cells increased $(42.4 \pm 5.3$ and $56.8 \pm 6.5$ for $1: 2$ and $1: 5$, respectively, $\mathrm{P}<0.05)$ and the mortality was more prominent than that of rTRAIL (32.1 $\pm 2.7, \mathrm{P}<0.05)$. For all the considered ratios, parental ADSCs did not induce A375 cell death $(9.4 \pm 1.1,10.2 \pm 1.3$ and $12.3 \pm 1.5$ for $1: 1,1: 2$ and $1: 5$, respectively), indicating specific action of TRAIL. To further confirm that A375 cell apoptosis is specifically due to the antitumor effect of TRAIL, anti-TRAIL was added to the coculture medium (Fig. 4C) and starting from $1 \mu \mathrm{g} / \mathrm{ml}(41.1 \pm 3.7)$, a significant $(\mathrm{P}<0.05)$ reduction of A375 cell death was identified. This effect was more prominent at the highest concentration $(35.3 \pm 4.2,28.2 \pm 3.3$ and $15.3 \pm 1.8$ for $2.5,5$ and $10 \mu \mathrm{g} / \mathrm{ml}$, respectively) where A375 death was comparable to that of the control group $(8.4 \pm 1.1$, $\mathrm{P}<0.05)$.

TRAIL-induces caspase activation. To further analyze the molecular events of TRAIL-induced cell apoptosis, A375 cells were indirectly co-cultured with TRAIL-ADSCs or parental ADSCs in a Transwell system for $48 \mathrm{~h}$, and then lysed to be subjected to western blot analysis. Fig. 5 shows that compared with parental ADSCs, the activity of caspase-4, -8, -9, and -3 in A375 cells was significantly induced in TRAIL-ADSC co-culture.

\section{Discussion}

Gene and targeted therapy is advancing rapidly and a large number of targeted therapies have been developed in the recent decade. However, current targeting agents exhibit frequent and severe toxicities as traditional cytotoxic agents (24). In the current study, ADSCs were used to induce TRAIL expression to mediate melanoma therapy. Such a modification has advantages compared with other strategies. For example, unlike DNA-based plasmid (pDNA) gene therapy $(25,26)$, TRAIL was first transfected into ADSCs. Our previous experiments modified 5'-cDNA ARCAs and 3'-poly(A) tails, which led to better transfection efficiency $(27,28)$ and translation into a functional protein in the cytoplasm directly. Furthermore, such a modification would not lead to any effect on the host genome and could be degraded by the host cell quickly (often within 2-3 days). Therefore, the risk of insertion mutagenesis is omitted.

In the current study, ADSCs were used as a carrier to deliver TRAIL protein, as the ADSCs express TRAIL protein which is then secreted into the extracellular space. Data showed that TRAIL protein was expressed in ADSCs and the active expression was retained for up to $72 \mathrm{~h}$ with the peak activity $48 \mathrm{~h}$ after transfection. These results indicated that the TRAIL protein synthesized in ADSCs was active and that the expression level is consistent with DNA-based vectors (3). The current study also demonstrated that TRAIL cDNA transfection did not alter the migration ability of ADSCs. Data further demonstrated that ADSCs migrated toward A375 cells compared with the HFFs, which indicated that ADSCs have tumor-oriented homing capacity. Thus, the current data provide a basis for the use of ADSCs in TRAIL-mediated anticancer therapy.

Furthermore, the TRAIL-ADSC antitumor activity in A375 cells was assessed and it was demonstrated that under co-culture conditions, TRAIL induced A375 cell apoptosis. This finding is consistent with previously published data on TRAIL activity $(8,21)$. The underlying molecular mechanism of TRAIL-ADSCs-mediated A375 cell death was also investigated and it was demonstrated that TRAIL-ADSCs altered the expression of members of the PI3K-AKT signaling pathway, including AKT, caspase-3, caspase-4, caspase- 8 and caspase-9. The phosphorylated form of AKT (pAKT) was markedly downregulated following co-culture with TRAIL-ADSCs. Caspase-3, caspase-4, caspase- 8 and caspase- 9 were activated after co-culture with TRAIL-ADSCs. Consistent with a study by Mao et al (13) 
A

$24 \mathrm{~h}$

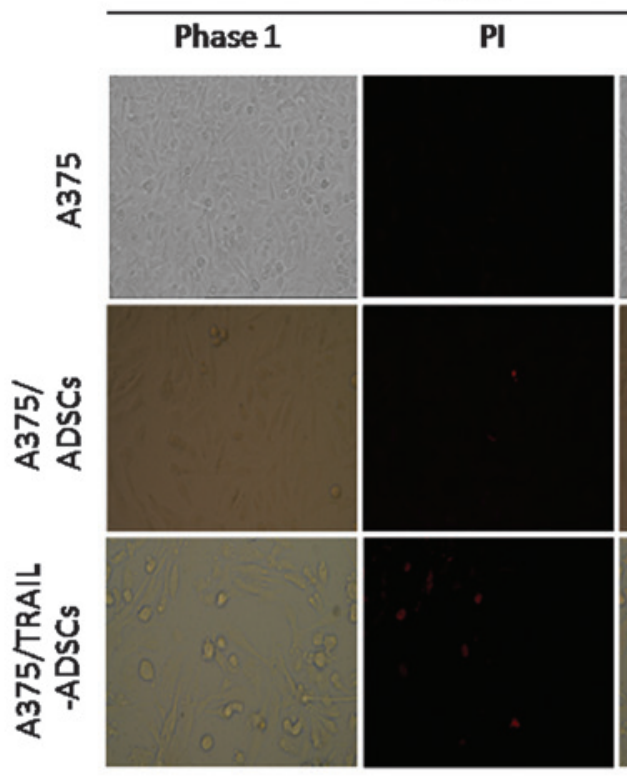

B

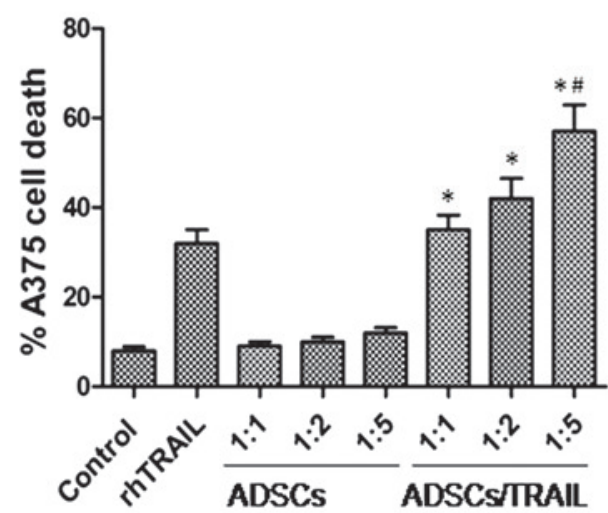

$48 \mathrm{~h}$

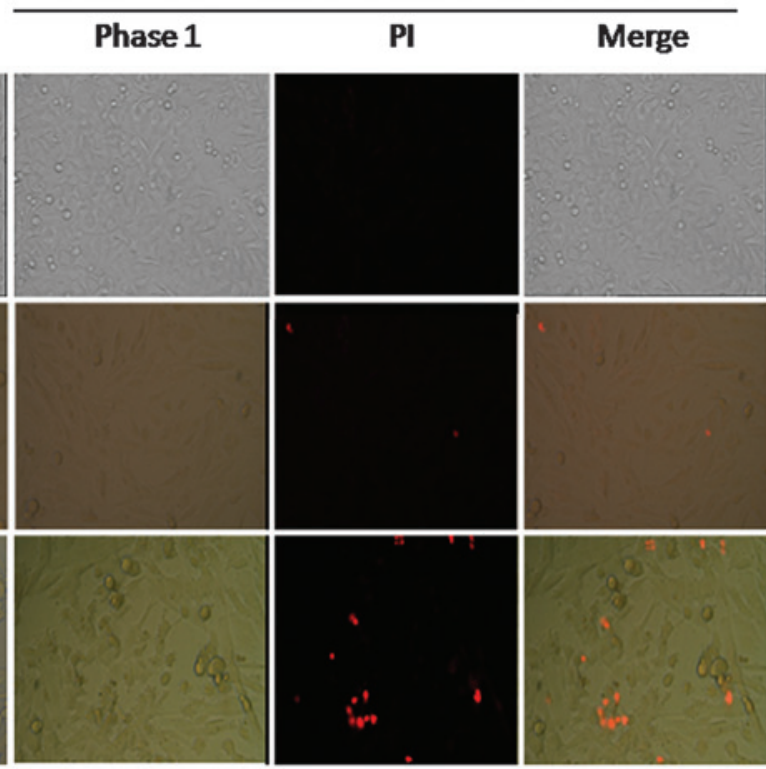

C

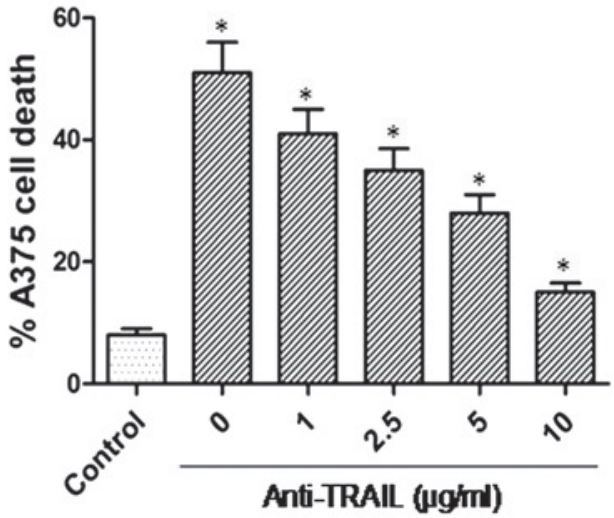

Figure 4. Induction of A375 cell apoptosis after indirect co-culture with TRAIL-ADSCs. (A) A375 cells were cocultured with ADSCs or TRAIL-ADSCs in a Transwell system, with A375 cells as control. After 24 or 48 h culture, the cells were stained with PI. Red staining indicates dead cells. Phase 1, whole population of cells that still attached to the culture surface assessed under the phase contrast microscope; PI, apoptotic cells stained with PI show red; merge, merged images of Phase 1 and PI. Original magnification, x100. (B) Fluorescence-activated flow cytometry was used to detect A375 cell apoptosis after staining with PI in co-culture with TRAIL-ADSCs or ADSCs at a different ratio in the Transwell system. rTRAIL served as a positive control and A375 cells alone served as a negative control. Ratio indicates the cell number of A375 cells to ADSCs or TRAIL-ADSCs. (C) Anti-human TRAIL antibody diluted at different concentrations was used to neutralize the TRAIL-induced apoptosis, which was shown in co-culture of A375 cells and TRAIL-ADSCs at a 1:1 ratio. ${ }^{*} \mathrm{P}<0.05$ vs. control, ${ }^{\#} \mathrm{P}<0.05$ vs. rhTRAIL. TRAIL, tumor necrosis factor-related apoptosis-inducing ligand; ADSCs, adipose-derived stem cells.
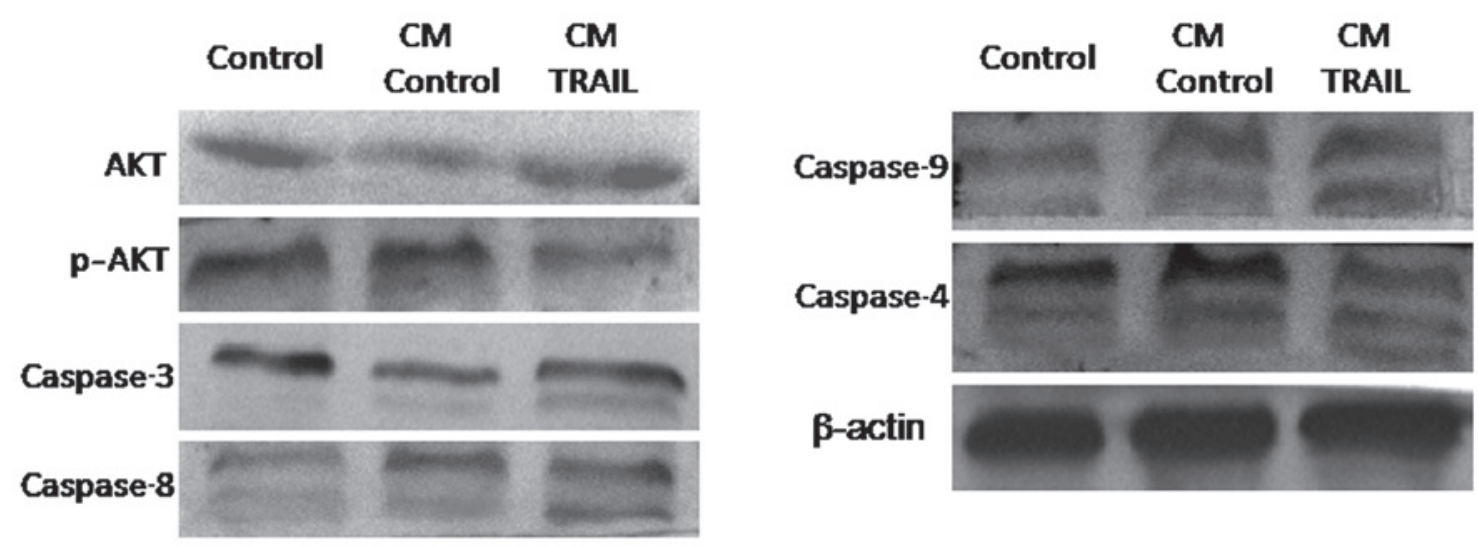

Figure 5. TRAIL-ADSCs mediated expression of the PI3K-AKT signaling genes in A375 cells after co-culture with TRAIL-ADSCs. Cells were harvested at $48 \mathrm{~h}$ after indirect co-culture with TRAIL-ADSCs and subjected to western blot analysis of PI3K-AKT signaling proteins. The multiple bands for the caspases indicate high activity. TRAIL, tumor necrosis factor-related apoptosis-inducing ligand; CM, conditioned medium; p-AKT, phosphorylated AKT; ADSCs, adipose-derived stem cells. 
TRAIL-induced apoptosis of human melanoma cells was observed to involve the activation of caspase-4. This appeared to be mediated by caspase- 3 , in that caspase- 4 was activated later than caspase-8, -9 and -3 , and that inhibition of caspase-3 blocked TRAIL-induced caspase-4 activation (13). These results indicated that the TRAIL-ADSCs-mediated melanoma cell death was associated the PI3K-AKT signaling pathway.

However, the current study was a proof-of-principle study and further investigation is required before this TRAIL-ADSCs system can be considered as a clinical strategy. Future studies will investigate the effects of TRAIL-ADSCs antitumor activity in vivo. In addition, investigation of the half-life or differentiation potential of TRAIL-ADSCs is also required. In conclusion, the strategy of this ADSC-mediated gene therapy may offer a double-targeted killing effect on tumor cells, consisting the tropism property of ADSCs and engineered anticancer agents. It is able to exert killing effects locally and consistently. This strategy also holds the potential for the use of the patient's own ADSCs and to switch tumor attackers corresponding to the individual situation. The present in vitro study provides an essential base for the use of synthesized mRNA in clinical trials. Further in vivo studies particularly combined with the use of clinical tumor samples may lead to development of a clinically meaningful patient-tailored anticancer therapy.

\section{References}

1. Vandamme N and Berx G: Melanoma cells revive an embryonic transcriptional network to dictate phenotypic heterogeneity. Front Oncol 4: 352, 2014

2. Stewart BW and Wild CP: World Health Organization: World Cancer Report 2014. Chapter 5.14. pp495-502.2014.

3. Jerant AF, Johnson JT, Sheridan CD and Caffrey TJ: Early detection and treatment of skin cancer. Am Fam Physician 62: 357-368, 375-376, 381-382, 2000.

4. Halachmi S and Gilchrest BA: Update on genetic events in the pathogenesis of melanoma. Cur Opin Oncol 13: 129-136, 2001.

5. Kanavy HE and Gerstenblith MR: Ultraviolet radiation and melanoma. Semin Cutan Med Surg 30: 222-228, 2011

6. Jost LM and ESMO Guidelines Task Force: ESMO minimum clinical recommendations for diagnosis, treatment and follow-up of cutaneous malignant melanoma. Ann Oncol 14: 1012-1013, 2003.

7. Balch CM, Soong SJ, Gershenwald JE, Thompson JF, Reintgen DS, Cascinelli N, Urist M, McMasters KM, Ross MI, Kirkwood JM, et al: Prognostic factors analysis of 17,600 melanoma patients: Validation of the American joint committee on cancer melanoma staging system. J Clin Oncol 19: 3622-3634, 2001.

8. Sun XY, Nong J, Qin K, Lu H, Moniri MR, Dai LJ and Warnock GL: MSC(TRAIL)-mediated HepG2 cell death in direct and indirect co-cultures. Anticancer Res 31: 3705-3712, 2011.

9. Loebinger MR and Janes SM: Stem cells as vectors for antitumour therapy. Thorax 65: 362-369, 2010.

10. Dai LJ, Moniri MR, Zeng ZR, Zhou JX, Rayat J and Warnock GL: Potential implications of mesenchymal stem cells in cancer therapy. Cancer Lett 305: 8-20, 2011.
11. Yang B, Wu X, Mao Y, Bao W, Gao L, Zhou P, Xie R, Zhou L and Zhu J: Dual-targeted antitumor effects against brainstem glioma by intravenous delivery of tumor necrosis factor-related, apoptosis-inducing, ligand-engineered human mesenchymal stem cells. Neurosurgery 65: 610-624, 2009.

12. Ivanov DP, Parker TL, Walker DA, Alexander C, Ashford MB, Gellert PR and Garnett MC: In vitro co-culture model of medulloblastoma and human neural stem cells for drug delivery assessment. J Biotechnol 205: 3-13, 2015.

13. Mao ZG, Jiang CC, Yang F, Thorne RF, Hersey P and Zhang XD: TRAIL-induced apoptosis of human melanoma cells involves activation of caspase-4. Apoptosis 15: 1211-1222, 2010.

14. Friedenstein AJ, Deriglasova UF, Kulagina NN, Panasuk AF, Rudakowa SF, Luriá EA and Ruadkow IA: Precursors for fibroblasts in different populations of hematopoietic cells as detected by the in vitro colony assay method. Exp Hematol 2: 83-92, 1974.

15. Zuk PA, Zhu M, Mizuno H, Huang J, Futrell JW, Katz AJ, Benhaim P, Lorenz HP and Hedrick MH: Multilineage cells from human adipose tissue: Implications for cell-based therapies. Tissue Eng 7: 211-228, 2001.

16. Zuk PA, Zhu M, Ashjian P, De Ugarte DA, Huang JI, Mizuno H, Alfonso ZC, Fraser JK, Benhaim P and Hedrick MH: Human adipose tissue is a source of multipotent stem cells. Mol Biol Cell 13: 4279-4295, 2002.

17. Kern S, Eichler H, Stoeve J, Klüter H and Bieback K: Comparative analysis of mesenchymal stem cells from bone marrow, umbilical cord blood, or adipose tissue. Stem Cells 24: 1294-1301, 2006.

18. Wiley SR, Schooley K, Smolak PJ, Din WS, Huang CP, Nicholl JK, Sutherland GR, Smith TD, Rauch C, Smith CA, et al: Identification and characterization of a new member of the TNF family that induces apoptosis. Immunity 3: 673-682, 1995.

19. Wu GS: TRAIL as a target in anti-cancer therapy. Cancer Lett 285: 1-5, 2009.

20. Rosenecker J, Huth S and Rudolph C: Gene therapy for cystic fibrosis lung disease: Current status and future perspectives. Curr Opin Mol Ther 8: 439-445, 2006.

21. Yamashita Y, Shimada M, Tanaka S, Okamamoto M, Miyazaki J and Sugimachi K: Electroporation-mediated tumor necrosis factor-related apoptosis-inducing ligand (TRAIL)/Apo2L gene therapy for hepatocellular carcinoma. Hum Gene Ther 13: 275-286, 2002.

22. Ashkenazi A, Pai RC, Fong S, Leung S, Lawrence DA, Marsters SA, Blackie C, Chang L, McMurtrey AE, Hebert A, et al: Safety and antitumor activity of recombinant soluble Apo2 ligand. J Clin Invest 104: 155-162, 1999.

23. Grisendi G, Bussolari R, Cafarelli L, Petak I, Rasini V, Veronesi E, De Santis G, Spano C, Tagliazzucchi M, Barti-Juhasz H, et al: Adipose-derived mesenchymal stem cells as stable source of tumor necrosis factor-related apoptosis-inducing ligand delivery for cancer therapy. Cancer Res 70: 3718-3729, 2010.

24. Dy GK and Adjei AA: Understanding, recognizing, and managing toxicities of targeted anticancer therapies. CA Cancer J Clin 63: 249-279, 2013.

25. Zangi L, Lui KO, von Gise A,Ma Q, Ebina W,Ptaszek LM, SpäterD, $\mathrm{Xu} \mathrm{H}$, Tabebordbar M, Gorbatov R, et al: Modified mRNA directs the fate of heart progenitor cells and induces vascular regeneration after myocardial infarction. Nat Biotechnol 31: 898-907, 2013.

26. Yoshioka N, Gros E, Li HR, Kumar S, Deacon DC, Maron C, Muotri AR, Chi NC, Fu XD, Yu BD and Dowdy SF: Efficient generation of human iPSCs by a synthetic self-replicative RNA. Cell Stem Cell 13: 246-254, 2013.

27. Leonhardt C, Schwake G, Stögbauer TR, Rappl S, Kuhr JT, Ligon TS and Rädler JO: Single-cell mRNA transfection studies: Delivery, kinetics and statistics by numbers. Nanomedicine 10: 679-688, 2014.

28. Stepinski J, Waddell C, Stolarski R, Darzynkiewicz E and Rhoads RE: Synthesis and properties of mRNAs containing the novel 'anti-reverse' cap analogs 7-methyl (3'-O-methyl) GpppG and 7-methyl (3'-deoxy) GpppG. RNA 7: 1486-1495, 2001. 